\title{
TERAPIAS COMPLEMENTARES: FITOTERAPIA COMO OPÇÃO TERAPÊUTICA NO CLIMATÉRIO E MENOPAUSA
}

\author{
Bruna Maria de Almeida Rochal \\ Maria do Socorro Vieira Pereira II \\ Jefferson Queiroz Carneiro III
}

\begin{abstract}
RESUMO
Climatério é a fase de vida da mulher associada à tristeza, sofrimento e envelhecimento, o que interfere de forma negativa na sua autoestima, uma vez que a beleza está interligada a juventude e a fertilidade, aspectos intensamente valorizados pela sociedade. $O$ estudo tem como objetivo realizar uma revisão sobre os aspectos biopsicossociais das mulheres em relação às alterações fisiológicas e psicológicas desta etapa da vida, o climatério e a menopausa, além de analisar terapêuticas alternativas, como a fitoterapia, que faz uso de plantas medicinais e utilização de fitormônios. Trata-se de uma revisão de literatura, pautando-se na publicação de referências encontradas em artigos indexados em base de dados e revistas científicas. Para a construção do estudo, consideram-se as pesquisas indexadas, na base de dados SCIELO - Scientific Eletronic Libray Online, periódico CAPES, bases de dados Medline, IBECS, utilizando-se descritores: mulher; climatério; aspectos biopsicossociais, fitoterapia. Os sintomas desenvolvidos e apresentados no climatério, estão geralmente relacionados, a deficiência hormonal particularmente, do estrógeno e progesterona. $O$ uso de plantas medicinais como Glycine Max L., a Cimicífuga racemosa L. Morus nigra L. são comumente utilizadas por mulheres nessa fase para reposição dos fitormônios, o que demonstra a relevância das práticas alternativas, como estratégia para o alívio dos sintomas, melhora e compreensão dessa fase como constituinte e integrante do seu ciclo de vida.
\end{abstract}

\section{PALAVRAS-CHAVE}

Mulher. Climatério. Aspectos biopsicossociais. Fitoterapia.

\section{INTRODUÇÃO}

O climatério é a fase da vida em que ocorre a transição do período produtivo ou fértil para o não reprodutivo, devido à diminuição dos hormônios sexuais produzidos pelos ovários. É um mecanismo interessante, pois a sexualidade ganha prioridades em relação à reprodução; enquanto a menopausa é um evento dentro do climatério e representa a última menstruação da vida da mulher. O climatério e a menopausa estão intrinsecamente ligados, ocorrendo em determinada fase na vida feminina, podendo trazer várias alterações, tanto psicológicas como fisiológicas ${ }^{1}$.

Atualmente, com a expectativa de vida da mulher brasileira, estimada hoje em 72 anos, calcula-se que a população

I Mestranda Programa de Pós-Graduação em Saúde da Família Mestrado Profissional em Saúde da Família FACENE, brunaenfe@hotmail.com

II Professora/Orientadora FACENE/FAMENE. Programa de Pós-Graduação em Saúde da Família Mestrado Profissional em Saúde da Família FACENE.

III Mestrando do Programa de Pós-Graduação em Saúde da Família - Mestrado Profissional em Saúde da Família da Faculdade de Enfermagem Nova Esperança - FACENE. 
feminina na fase da menopausa, período que constitui um terço da sua existência, seja mais de cinco milhões no país. Essas passaram a viver mais tempo, vivenciando modificações em seus corpos que outras gerações não conseguiram. O climatério, que integra esse processo, é considerado um evento natural, ocorrendo em função da falência ovariana podendo ou não apresentar sintomas denominados de síndrome climatérica 2,3.

$\mathrm{Na}$ atualidade, com o aumento da longevidade e a manutenção da idade da menopausa, a mulher poderá passar mais de um terço de sua vida, após o climatério, o que implica na preocupação com o tratamento dos sintomas que acompanham esse período das comorbidades, associadas ao envelhecimento, de importância crescente na saúde das mulheres, objetivando melhor qualidade de vida 4.

Embora a menopausa, assim como o próprio envelhecimento, seja um evento fisiológico, a diminuição do estrógeno e da progesterona, decorrente, associa-se a inúmeras repercussões negativas sobre o organismo feminino. Os principais problemas que são apresentados pelas mulheres no climatério são: irregularidades menstruais, sintomas vasomotoras, insônia, instabilidade do humor, atrofia urogenital, osteoporose, doenças cardiovasculares, câncer e problemas cognitivos 5 .

O Sistema Público de Saúde no Brasil não possui uma política de assistência farmacêutica capaz de suprir a demanda medicamentosa da população, sobretudo no nordeste brasileiro, onde a população carente apresenta problemas para obter os medicamentos essenciais, bem como adoece muito mais. Com a descentralização do poder público, atualmente em nosso país, o município atinge a gestão plena, com liberdade para implantar programas de assistência à saúde, quando necessários. Desta forma, alguns estados e municípios brasileiros vêm realizando nas duas últimas décadas a implantação de programas de fitoterapia na atenção primária à saúde, com o objetivo de suprir as carências medicamentosas de suas comunidades ${ }^{6}$.

A utilização de plantas medicinais é uma ferramenta importante para profissionais de saúde, usuários, pesquisadores e gestores, o que levou o Ministério da Saúde a criar a Política Nacional de Práticas Integrativas e Complementares no SUS. Posteriormente, também foram criados a Política Nacional de Plantas Medicinais e Fitoterápicos e o Programa Nacional de Plantas Medicinais e Fitoterápicos 7 .

A terapia de reposição hormonal é muito questionada por mulheres que fazem uso desse processo terapêutico, o que faz muitas delas buscarem uma alternativa segura. Existem várias alternativas à terapia de reposição hormonal para aliviar os sintomas da menopausa. A literatura descreve que Cimicifuga racemosa (L.), uma nova combinação para Actaea reacemosa L., teve efeitos benéficos nos fogachos em mulheres no climatério, sendo uma das plantas mais estudadas e aplicadas nestes casos, por apresentar alternativa mais segura para mulheres no climatério que demonstram contraindicações à terapêutica hormonal clássica 8,9.

Os sintomas relacionados com as atitudes e comportamento são sintomas subjetivos, como alterações de humor ou depressão. A literatura afirma que os fogachos e outros sintomas agudos associados ao período da perimenopausa, frequentemente, se tornam mais intensos próximos da menopausa, quando os níveis de estrogênio circulante caem subitamente. Para alívio ou eliminação desses sintomas, é indicada a reposição hormonal. No entanto, muitos estudos foram intensificados para a averiguação científica do efeito de uma alimentação 
balanceada e do uso de concentrados dos fitoestrogênios contra os efeitos da menopausa, destacando-se a utilização de uma reposição hormonal por meios alternativos e naturais ${ }^{10}$.

A utilização da fitoterapia é uma prática de grande aceitação popular e que envolve vários profissionais da área da saúde e com a implementação da Política Nacional de Plantas Medicinais e aos Fitoterapêuticas (PNPM), aprovada por meio do Decreto $n^{\circ}$ 5.813. A população brasileira conquistou avanços importantes relacionando a fitoterapia ${ }^{11}$. Acreditar na naturalidade inócua, dos fitoterápicos e plantas medicinais não é facilmente contradita, pois as evidências científicas de ocorrência de intoxicações e efeitos colaterais, relacionados com o uso de plantas medicinais, consistem em informações que dificilmente chegam ao alcance dos usuários atendidos nos serviços de saúde pública, caracterizado como indivíduos de baixa escolaridade e acervo cultural ${ }^{12,13 .}$

Estudos, realizados por Petry e Ramon Junior (2012) ${ }^{14}$, sobre a viabilidade de implantação de fitoterápicos, em um município do Rio Grande do Sul, demonstraram que $98 \%$ dos entrevistados possuíam interesse nessa classe de medicamentos. O que confirma que a população recorre a protocolos terapêuticos alternativos como a fitoterapia, principalmente, devido à consciência ecológica e a crença popular de que o natural é inofensivo. Isto é reforçado pelos efeitos indesejáveis e prejuízos causados pelo uso abusivo e/ou incorreto dos medicamentos sintéticos, a falta de acesso aos medicamentos e à medicina institucionalizada 15,16 .

No período do climatério e, prin- cipalmente, no período pós-menopausa ocorrem alterações fisiológicas em todo organismo; eixo neuroendócrino, reprodutor, sistema cardiovascular, geniturinário; ossos e pele. Os sintomas identificados sofrem influência de inúmeros fatores de ordem biológica, aspectos psicológicos e aspectos socioculturais, integrados aos mitos, crenças e preconceitos que a sociedade constitui, dissemina e vivencia em cada época ${ }^{2}$.

O aumento da expectativa de vida traz um incremento do número de mulheres climatéricas que necessitam de atenção da equipe de saúde, com orientações simplificadas, através de normas do consenso para o atendimento da vida. Atualmente, as mulheres apresentam uma preocupação maior com as mudanças que ocorrem com o seu corpo, no intuito de obter uma melhor qualidade de vida. O que significa ficar livre dos sintomas do climatério que prejudicam seu bem-estar geral. A assistência à mulher no climatério, incluindo a sexualidade, é uma das prioridades das Políticas Públicas de Saúde tornando evidente a necessidade de atenção nesta área, que caracteriza o término do período reprodutivo ${ }^{1}$.

Diante do exposto, a referida pesquisa tem como objetivo realizar uma revisão sobre os aspectos biopsicossociais das mulheres, em relação às alterações fisiológicas e psicológicas, nessa etapa da vida, e a busca de terapêuticas alternativas, como a fitoterapia, através do uso de plantas medicinais e utilização de fitormônios. O estudo é de relevância para a compreensão e conhecimento das ações terapêuticas alternativas no climatério e menopausa, pois também visa a melhoria da qualidade de vida dessas mulheres. 


\section{MATERIAIS E MÉTODO}

Foram realizadas buscas de produção literária científica para a compreensão do conhecimento sobre os aspectos biopsicossociais e o uso de terapias complementares, particularmente a fitoterapia e fitomedicamentos, por mulheres na fase de climatério. Para a construção

\section{RESULTADOS E DISCUSSÃO}

A Organização Mundial da Saúde (OMS) considera que o climatério, ou Peri menopausa, chega ao término um ano depois da menopausa. A pré-menopausa é o período de tempo que se iniciam com o aparecimento dos sintomas climatérios, ou seja, ondas de calor ou irregularidades menstruais, quase sempre a partir dos 45 anos 17,18 .

A diminuição dos níveis hormonais é um fato que ocorre com todas as mulheres e se inicia ao redor dos 40 anos. Algumas mulheres podem apresentar um quadro mais acentuado de sinais e sintomas, porém todas chegarão à menopausa. A diminuição ou a falta dos hormônios sexuais feminino podem afetar vários locais do organismo e determinam sinais e sintomas conhecidos pelo nome de síndrome climatérica 19.

No climatério, os ovários passam a não responder à estimulação da hipófise acarretando alterações hormonais e ocorrendo a elevação de gonadotrofinas pituitárias, de hormônios luteinizantes e de hormônios estimulantes foliculares. Consequentemente, há um decréscimo na produção de estrogênio pelo ovário e perda da regeneração e inibição do nível hipotálomopituitário. Como resultado, a diminuição de estrógeno leva a alterações metabólicas, sinais clínicos e sintomas do climatério em vários níveis: morfológico, hormonal, metabólico, funcional, psíquico e cognitivo 20.

O número de folículos ovarianos do estudo, consideraram-se as pesquisas indexadas, na base de dados SCIELO - Scientific Eletronic Libray Online, periódico CAPES, bases de dados Medline, IBECS, utilizando-se os descritores: mulher. Climatério. Aspectos biopsicossociais. Fitoterapia.

disponíveis torna-se cada vez menor e os remanescentes ficam menos responsivos as gonadotrofinas hipofisárias FSH e LH. Com a redução do estradiol deixa de ocorrer o feedback negativo que estes exerciam no eixo hipotálamo-hipófiso-ovariano que era desencadeado por este tipo de hormônio 17 .

Na mulher, a mudança mais perceptível é a cessação espontânea do ciclo menstrual, o que se denomina menopausa. Para considerar uma mulher menopáusica, a menstruação deve ter cessado durante um ano. Estabelecida a menopausa, a mulher chega ao término do seu período reprodutivo, durante os anos que precedem à menopausa, os ciclos tornam-se irregulares ${ }^{21}$.

A mulher climatérica, que apresenta um estado emocional equilibrado, procura buscar uma qualidade de vida melhor, enfrentando com naturalidade os acontecimentos desta fase e sente-se gratificada e realizada, com tudo que diz respeito a este momento de sua existência. Contrapõe-se as que apresentam um estado emocional instável, afetado por pensamentos e sentimentos negativos, apresentando uma percepção de vida repleta de perdas com experiências vividas não muito boas, passando a ter uma visão errônea da fase, enfrentando com dificuldade este período ${ }^{18}$.

A sintomatologia do climatério está diretamente ligada à deficiência estrogênica e a sua intensidade geralmente 
é proporcional ao nível dessa deficiência. Se a queda dos estrogênios circulantes ocorre, como nos casos de remoção cirúrgica ou irradiação dos ovários, os sintomas costumam ser intensos e de manifestação quase imediata. Quando a queda da função ovariana ocorre de maneira mais lenta e progressiva, os sintomas são mais leves, às vezes até imperceptíveis. Entre esses extremos, existe um amplo espectro de sintomatologia, variável de uma mulher para outra e sujeita a condicionamentos socioculturais e psíquicos 4 .

Comumente para algumas mulheres, o climatério passa despercebido, isto é, sem sintomas, o que não significa que elas estejam isentas de suas consequências. Os sintomas são os que caracterizam a síndrome do climatério. Os aspectos culturais e psíquicos são importantes no agravo sintomatologia. Destacam-se entre eles medo de envelhecer, preocupação com autoimagem, cada vez mais sentida na sociedade moderna, instabilidade conjugal, síndrome do ninho vazio e competição com o marido 22 .

Muitas mulheres relatam sintomas psicológicos durante o climatério, apesar de ser discutível se estes são causados especificamente pela deficiência de estrogênios, ou na realidade, se são consequências de outros problemas, tais como a falta de sono, fatores sociais ou exaltação de problemas psicológicos e psiquiátricos subjacentes. Algumas experimentam ansiedade e depressão mas, geralmente, a estrutura da personalidade e pré-menopausa de uma mulher a predispõe à síndrome da pré-menopausa 23.

A pele é afetada pela privação de estrogênios, bem como pelas alterações gerais relacionada com a idade, facilidade para formar equimoses, ressecamento, quedas de cabelo e unhas quebradas, fibras elásticas e colágenos se degeneram, glândulas sebáceas e sudoríparas tornam-se menos ativas e vasos sanguíneos evidenciam sinais de esclerose. A dis- tribuição de pelos também se modifica, ocorre aumento na face e redução da purificação pubiana, axilar e dos cabelos no couro cabeludo, causado pelo aumento da relação androgênio e estrogênio. Essa condição é importante para autoestima e autoconfiança da mulher, observa-se que as alterações pós-menopausa na pele e cabelo afetam a qualidade de vida 19 .

A sintomatologia, apresentada pela mulher no climatério, varia de acordo com a estrutura emocional e com o estilo de vida que cada uma teve até então. Se a mulher for estruturada psicologicamente, se for realizada em sua vida profissional, familiar e sexual, poderá enfrentar mais facilmente os transtornos causados nesse período. Assim, o climatério poderá ser um tempo de novas experiências e conquistas 17 .

\section{Aspectos biopsicossociais femini- no no climatério}

A mulher na fase do climatério sofre transformações afetando suas emoções, seu corpo e seu comportamento, com as pessoas que vivem ao seu redor. A ideia do envelhecer é para algumas delas uma época de perdas, um período de psicoinstabilidade quando mudanças ocorrem lentamente e começam a ser notadas no dia-a-dia como, por exemplo, alguns esquecimentos, tristezas, entre outros. No que concerne aos sintomas depressivos, constata-se que a mulher deprimida sentese num sombrio estado de ânimo, descrevendo como tristeza e demonstrando perda de energia e interesse, sentimento de culpa, pensamentos de morte, bem como alterações do nível de atividades habituais como: apetite, atividade sexual e sono 4. Dados epidemiológicos sobre menopausa e sintomas climatéricos são raros no Brasil. Luis Filho e outros autores ${ }^{20}$ realizaram estudo recente que demonstrou, que mulheres com idade à menopausa inferior a 46,6 anos apresentam risco $16 \%$ maior de morte por qualquer causa. Observa-se que 
a mortalidade específica por causas coronarianas, respiratórias, geniturinárias; o que pode ser explicado pela deterioração precoce de células vasculares endoteliais, células de músculo liso e do miocárdio que apresentam receptores sensíveis à ação do estrogênio.

A falta de conhecimento, sobre as fases de climatério e menopausa, poderá tornar essas mulheres incapazes de expressar o que sentem, guardando para si seus momentos de dúvidas e incertezas. A principal atividade profilática do climatério é promover o esclarecimento e o autocuidado, pois, uma pessoa que conhece o seu próprio corpo, terá mais condições de se comportar de modo mais saudável, sem falsas expectativas, tabus ou receios fantasiosos.

\section{Abordagem sobre as opções terapêu- ticas no climatério emenopausa}

$\mathrm{Na}$ terceira fase da vida, a mulher começa a sofrer uma perda hormonal, isso ocorre por volta dos 40 anos de idade, quando começa o período do climatério. A queda hormonal que ocorre no período do climatério apresenta em muitas mulheres diversos sintomas, como as ondas de calor, suores noturnos, insônia, depressão, irritabilidade, ressecamento vaginal, redução no desejo sexual, dores no momento do ato sexual, dentre outros 24 .

A finalidade da terapia de reposição hormonal é suprir a falta de hormônios; que poderá utilizar a forma convencional ou tradicional em que se faz o uso de medicamentos sintéticos e a alternativa através de fitoestrôgenos. Estudos relataram que as mulheres que faziam uso de hormônios sintéticos apresentaram maior incidência na formação de células cancerígenas. Sendo assim, inúmeros estudos estão sendo realizados como terapia alternativa, através de alimentos, e em especial as que usam as isoflavonas da soja como fonte natural de fito-hormônios ${ }^{10}$.

Levando-se em conta a busca pela manutenção da saúde de forma natural, uma área promissora no desenvolvimento de terapias fitoterápicas, tange ao tratamento dos sintomas do climatério, haja vista que o protocolo atual com reposição hormonal, através de fito-hormônios, tem sido considerado um meio alternativo no tratamento dos sintomas apresentados por mulheres nessa fase da vida. A soja, por exemplo, é uma importante fonte desses compostos e tem sido associada à redução da frequência e intensidade dos fogachos, sintoma recorrente no climatério 25 .

Nos últimos anos, os fito-hormônios, substâncias de origem vegetal com características hormônios-like, surgiram para substituir a terapia de reposição hormonal, o que se deve ao anúncio dos riscos associados à terapia de reposição hormonal para mulheres no climatério no ano de 2002, e uma mudança de percepção do público consumidor e laboratórios farmacêuticos a respeito das plantas medicinais 26 .

A inclusão da fitoterapia na Atenção Básica tem sido discutida em diversas oportunidades no Brasil, como em

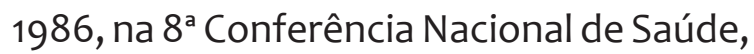
quando foi recomendada a introdução das práticas tradicionais de cura popular no atendimento público de saúde 27 .

$\mathrm{Na}$ atualidade, a legislação brasileira passou a incentivar a busca por novas alternativas terapêuticas e, inclusive, já determina uma relação de medicamentos essenciais a própria soja voltada para o tratamento de sintomas do climatério. Estudos têm demonstrado que o Trifolium pratense e a Cimicífuga racemosa, que podem ser utilizados nesta fase da vida, já são indicadas pelo Manual de Atenção a Mulher no Climatério, um importante documento que subsidia a prescrição de fito- 
terápicos na atenção primária 28.

\section{Plantas utilizadas no tratamento de sintomas do climatério}

Segundo Brasil (2008b) ${ }^{29}$, os principais fitoterápicos utilizados são comumente fonte de fitoestrogênios por sua ação estrogênio-símile, sendo os mais utilizados o Glycine max, Trifolium pratense e a Cimicífuga racemosa, apesar de existirem muitos outros fitoterápicos com esta finalidade. Existem ainda medicação fitoterápica específica para alívio de quadros leves e moderados de depressão e ansiedade, utilizados também em cardiologia, neurologia, psiquiatria, entre outros. Para os sintomas psicoemocionais, que podem acompanhar esta fase da vida da mulher, é válido ressaltar o uso de Hiperico perforatum, Valeriana officinalis, Melissa officinalis. No Canadá, segundo ROWE (2014), estão regulamentadas Glycine max, Hypericum perforatum (sintomas da depressão), Dioscorea villosa (reduz sintomas da menopausa), Cimicifuga racemosa (reduz fogachos), coincidindo com duas plantas da

\section{CONSIDERAÇÕES FINAIS}

O período do climatério é identificado por inúmeras transformações e marcado pelo término do período reprodutivo. As mudanças nos aspectos biopsicossociais são expressivas e uma vez conduzidas de maneira inapropriada, podem prejudicar a qualidade de vida e o bem-estar psicológico. Os aspectos culturas e psíquicos são os mais importantes no agravo da manifestação dos sintomas como, por exemplo, o medo de envelhecer, a preocupação com autoimagem, cada vez mais sentida na sociedade moderna e instabilidade conjugal, o que altera de forma significativa o dia-a-dia da mulher.

Os sintomas decorrem devido à baixa de estrogênio e progesterona san- legislação brasileira.

Pesquisas atuais de Schiavo com outros autores 30 demonstram, após entrevistar 84 mulheres com idade média de 51,6 anos no município de Três Passos/RS, a utilização de duas plantas medicinais voltadas para diminuir os calores e outros sintomas: Morus alba L. (Amoreira branca) e Calendula officinalis L. (Calêndula). Esses resultados indicam que as plantas medicinais podem ser consideradas um recurso para o auxílio do tratamento do climatério.

A C. racemosa é planta nativa do leste da América do Norte, onde é conhecida como Black cohosh, sendo conhecida no Brasil como cimicífuga ${ }^{28}$. Os extratos dessa planta foram usados historicamente como anti-inflamatórios, antipiréticos e analgésicos, entre outras indicações, como no tratamento das cólicas menstruais e nos sintomas da menopausa. Recentemente, passou a ser utilizado em todo mundo mas, principalmente, nos Estados Unidos e na Europa, por mulheres para aliviar os sintomas do climatério 31, 9 .

guíneo, o que causa alterações teciduais nos órgãos genitais, as quais geram não apenas desconforto físico como também psicológico. Na busca do alívio dos sintomas e a partir do conhecimento dos efeitos colaterais da terapia de reposição hormonal, muitas mulheres recorrem a terapia alternativa usando como tratamento, os fitoterápicos. Também se faz uso de fito-hormônios, seja a partir da prescrição de formulação, a base de soja, ou da introdução desta, na dieta da mulher.

A legislação brasileira passou a incentivar nos últimos anos, a busca por novas alternativas criando a Relação de Medicamentos Essenciais, indicados pelo Manual de Atenção a Mulher no Cli- 
matério30, um importante documento que subsidia a prescrição de fitoterápicos na atenção primária.

Os fito-hormônios e o uso das plantas medicinais representam um recurso alternativo para o tratamento e alívio dos sintomas no climatério. São raras as pesquisas nessa área. É necessário o desenvolvimento e investigação científica, uma vez que as pesquisas atuais demonstram um pequeno número de plantas medici- nais direcionadas ao climatério, o que evidencia também a busca de fomentar, não apenas a capacitação de profissionais de saúde, para a prescrição de fitoterápicos e instigar a comunidade acadêmica a investigar a eficácia, qualidade e segurança de antigas e novas preparações fitoterápicas. Também melhorar a qualidade de vida das mulheres nessa fase, como estratégia para o alívio dos sintomas, melhora e compreensão do seu ciclo de vida.

\title{
COMPLEMENTARY THERAPIES: PHYTOTHERAPY AS A THERAPEUTIC OPTION IN THE CLIMACTERIC AND MENOPAUSE
}

\begin{abstract}
Climacteric is the life stage of the woman associated with sadness, suffering and aging, which interferes in a negative way in her self-esteem, since beauty is intertwined with youth and fertility, which is intensely valued by society. The objective of this study is to review the bio psychosocial aspects of women in relation to the physiological and psychological changes of this stage of life in the climacteric and menopause, and the search for alternative therapies, such as herbal medicine through the use of medicinal plants and the use of phytohormones. It is a literature review, based on the publication of references found in articles indexed in databases and scientific journals; for the construction of the study, we consider the indexed surveys, in the SCIELO database - Scientific Electronic Library Online, CAPES journal, Medline databases, IBECS, using descriptors: Woman; climacteric; biopsychosocial aspects, phytotherapy. The symptoms developed and presented in climacteric, are generally related, hormonal deficiency in particular, of estrogen and progesterone, the use of medicinal plants such as Glycine Max L., Cimicífuga racemosa L. Morus nigra L. are commonly used by women at that stage to which demonstrates the relevance of alternative practices as a strategy for symptom relief, improvement and understanding of this phase as a constituent and integral part of its life cycle.
\end{abstract}

\section{KEYWORDS}

Woman. Climacteric. Biopsychosocial aspects. Phytotherapy.

\section{REFERÊNCIAS}

1 Alves ERP, Costa AM, Bezerra SMMS, Nakano AMS, Cavalcanti AMTS, Dias MD. Climatério: $A$ intensidade dos sintomas e o desempenho sexual. Texto Contexto Enferm, Florianopolis, 2015; Jan-Mar; 24(1): 64-71.

2 Silva AR, Ferreira, TF, Tanaka ACA. História ginecológica e sintomatologia climatérica de mulheres pertencentes a uma unidade de saúde pública do Estado do Acre. Journal of Human Growth and
Development, 2010; 20(3): 778-786.

3 Zanette VC, Rossato AE, Citadini-Zanette $V$, Bernardi $F B C$. Prevalência do uso de fitoterapia para alívio de sintomas apresentados em pacientes climatéricas. Arquivos Catarinenses de Medicina, 2011; 40(1): 12-17.

4 Valença CN, Nascimento Filho JM, Germano RM. Mulher no Climatério: Reflexões sobre Desejo Sexual, Beleza e 
Feminilidade. Revista Saúde e Sociedade, 2010; 19(2):273-85.

5 Sogimig. Manual de Ginecologia e Obstetrícia. 5. ed. Belo Horizonte: Coopmed; 2012.

6 Silva MIG, Gondim APSG, Nunes IFS, Sousa FCF. Utilização de fitoterápicos nas unidades básicas de atenção à saúde da família no município de Maracanaú (CE). Revista Brasileira de Farmacognosia, 2006; Out./Dez; 16(4): 455-462.

7 Brasil. Ministério da Saúde. Secretaria de Ciência, Tecnologia e Insumos Estratégicos. Departamento de Assistência Farmacêutica e Insumos Estratégicos. Programa nacional de plantas medicinais e fitoterápicos. Brasília, 2009. 135 p., il. (Série C. Projetos, Programas e Relatórios).

8 Radowicki S. et al. Skutecznosc i bezpieczenstwo stosowania suchego wyciagu z klacza Cimicifuga racemosa w leczeniu objawów zespolu menopauzalnego. Ginekologia Polska, 2006; 77(9):678-83.

9 Silva AG, Brandao AB, Cacciari RS, Soares WH. Avanços na elucidação dos mecanismos de ação de Cimicífuga racemosa (L.) Nutt. nos sintomas do climatério. Rev. Bras. Plantas med., 2009; 11(4).

10 Carvalho MAPF, Costa JFO. Derivados Vegetais Similares a Estrógenos (Dvse) no Tratamento dos Sintomas do Climatério. Revista Fitos, 2013; 6(1).

11 Brasil. Ministério da Saúde. Secretaria de Ciência, Tecnologia e Insumos Estratégicos. Departamento de Assistência Farmacêutica. A fitoterapia no SUS e o Programa de Pesquisa de Plantas Medicinais da Central de Medicamentos/ Ministério da Saúde, Secretaria de Ciên- cia, Tecnologia e Insumos Estratégicos, Departamento de Assistência Farmacêutica. - Brasília: Ministério da Saúde, 2006.

12 Alexandre RF, Bagatini F, Simões CMO. Potenciais interações entre fármacos e produtos à base de valeriana ou alho. Rev Bras Farmacogn 2008;18:455-463.

13 Silva MIG 2003. Utilização de Fitoterápicos nas Unidades Básicas de Saúde da Família (UBSF) no Município de Maracanaú-CE. Fortaleza, 144p. Dissertação de Mestrado - Faculdade de Farmácia, Odontologia e Enfermagem, Universidade Federal do Ceará.

14 Silveira, PF, Bandeira MA, Arrais PSD. Fármaco vigilância e reações adversas às plantas medicinais e fitoterápicos: uma realidade. Rev. bras. farmacogn. [online]. 2008;18(4):618626. Available from: <http://www. scielo.br/ scielo.php?script=sci_arttext\&pid=S0102-695X2008000400021\&Ing=en\&nrm=i so $>$.

15 Petry K, Ramon-Junor W A. Viabilidade de implantação de fitoterápicos e plantas medicinais no Sistema Único de Saúde (SUS) do município de Três Passos/RS. Revista Brasileira de Farmacologia, 2012; 1 (93): 60-67.

16 Joly CA, Haddad CFD, Verdade LM, Oliveira MZ, Bolzani VS, Berlinck RGS. Diagnóstico da pesquisa em biodiversidade no Brasil. Revista USP, 2011; (89): 114-133.

17 Oliveira LAR, Machado RD, Rodrigues AJL. Levantamento sobre o uso de plantas medicinais com a terapêutica anticancer por pacientes da Unidade Oncológica de Anápolis. Rev. Bras. PI. Med., Campinas, 2014; 16(1): 32-40. 
18 Halbe, Hans Wolfgang. et al. Tratamento de reposição hormonal no climatério e na pós-menopausa In: Carvalho Filho, ET, Papaléo Netto M. Geriatria: Fundamentos, clínica e terapêutica. 2. ed. São Paulo: Athneu; 2006, p. 415.

19 Freitas ER, Barbosa AJG. Qualidade de vida e bem-estar psicológico no climatério. Arq. Bras. Psicol., 2015; 67(3).

20 Filho, JFL, Baccaro LFC, Fernandes T, Conde DM, Paiva LC, Neto AMP. Epidemiologia da menopausa e dos sintomas climatéricos em mulheres de uma região metropolitana no sudeste do Brasil: inquérito populacional domiciliar. Rev. Bras. Ginecol. Obstet., 2015; 37(4): 152-8.

21 Santos, ÁAS, Silva FV, Martins FL. Percepção das mulheres no município de Paulo Afonso, na Bahia, sobre as mudanças corporais e emocionais no período do climatério. Estação Científica UNIFAP, Macapá, 2016; Jan./Abr. 6(1); 91-104. 22 Poli MEH, Schwanke CHA, Cruz IBM. A menopausa na visão gerontológica. Scientia Médica, 2010; 20(2):176-184.

23. De Lorenzi, DRS, Catan LB, Moreira K, Ártico GR. Assistência à mulher climatérica: novos paradigmas. Revista Brasileira de Enfermagem, 2009; 62(2):287-293.

24 Martins, MAD, Nahas EAP, Nahas-Neto J, Uemura G, Buttros DAB, Traiman P. Qualidade de vida em mulheres na pós-menopausa, usuárias e não usuárias de terapia hormonal. Revista Brasileira de Ginecologia e Obstetrícia, 2009; 31(4): 196-202.

25 Anjo, MRAS. Menopausa em Revista: discursos praticados pela revista Maria em torno da menopausa. 2010. 224 f. Dissertação - Universidade Aberta de Lisboa, Lisboa, 2010.

26 Livinalli A, Lopes LC. Avaliação das prescrições de isoflavonas para mulheres no climatério em cidade de médio porte do estado de São Paulo. Revista de Ciências Farmacêuticas Básica e Aplicada, 2007; 28: 185-191.

27 Sá, IM. “Fito-hormonios": ciência e natureza no tratamento do climatério. Phisis, 2012; 22(4).

28 Brasil. Ministério da Saúde. Anais da Conferência Nacional de Saúde, n. 8. Brasília: Ministério da Saúde; 1987.

29 Brasil, Ministério da Saúde. Secretaria de Atenção à Saúde. Departamento de Atenção Básica. Práticas Integrativas e Complementares em saúde: uma realidade no SUS. Revista Brasileira Saúde da Família, Brasília, v. 9, n. especial, p. 70-76, maio, 2008a.

30 Brasil. Ministério da Saúde. Secretaria de Atenção à Saúde. Departamento de Ações Programáticas Estratégicas. Manual de Atenção à Mulher no Climatério/Menopausa. Brasília: Ministério da Saúde, 2008b.

31 Schiavo M, Colet CF, Cavalheiro CAN, Molin GTD, Cavinatto AD, Schwambach MKP, Oliveira, KO. Avaliação do uso de plantas medicinais por mulheres residentes em Ijuí/RS. Revista Brasileira de Medicina na Família, 2015; 10(16).

32 Bolle, P. et al. Estrogen-like effect of a Cimicifuga racemosa extract sub-fraction as assessed by in vivo, ex vivo and in vitro assays. Journal of Steroid Biochemistry and 